\title{
On RAM Finite Hyperbolic Transforms
}

\author{
R.A.Muneshwar ${ }^{1}$, K.L.Bondar ${ }^{2}$, V.S.Thosare ${ }^{3}$ \\ ${ }^{I}$ Department of Mathematics, NES Science College, SRTMU Nanded, India - 431602 \\ ${ }^{2}$ Department of Mathematics, NES Science College, SRTMU Nanded, India - 431602 \\ ${ }^{3}$ Department of Mathematics, NES Science College, SRTMU Nanded, India - 431602
}

Abstract: In this paper we have introduced the new concept of RAM finite hyperbolic transforms. Transform of some standard functions are obtained and some properties are proved.

Keywords: Generalized Transform, Finite transform, RAM Finite hyperbolic transform, Transform of some standard functions.

\section{Introduction:}

The Laplace transform method is normally used to find the response of a linear system at any time to the initial data at $\mathrm{t}=0$ and disturbance $\mathrm{f}(\mathrm{t})$ acting for $\mathrm{t}>0$. If the disturbance is $\mathrm{f}(\mathrm{t})=e^{a t^{2}}$, for a $>0$, the usual Laplace transform cannot be used to find the solution of an initial value problem because Laplace transform of $\mathrm{f}(\mathrm{t})$ does not exist. It is often true that the solution at times later than $t$ would not affect the state at time $t$. This leads to define Finite Laplace transform.

The finite Laplace transform of a continuous or an almost piecewise continuous function $f(t)$ in $(0, T)$ is denoted by $\mathrm{L}_{\mathrm{T}}(\mathrm{f}(\mathrm{t}))=\mathrm{F}(\mathrm{p}, \mathrm{T})$, and is defined by

$\mathrm{L}_{\mathrm{T}}(\mathrm{f}(\mathrm{t}))=\mathrm{F}(\mathrm{p}, \mathrm{T})=\int_{0}^{\mathrm{T}} \mathrm{f}(\mathrm{t}) \mathrm{e}^{-\mathrm{pt}} \mathrm{dt}$

Where $\mathrm{p}$ is a real or complex number and $\mathrm{T}$ be a finite number which may be positive or negative.

Note : Above definition is defined for any bounded interval $\left(-\mathrm{T}_{1}, \mathrm{~T}_{2}\right)$.

Finite Laplace transform motivate us to define RAM Finite Sine Hyperbolic transform and RAM Finite Cosine Hyperbolic transform in $0 \leq \mathrm{t} \leq \mathrm{T}$ in order to extend the power and usefulness of usual Laplace transform in $0 \leq$ $t<\infty$. In section 2, the concept of RAM Finite Hyperbolic Transforms is introduced. Section 3 is devoted to explain existence conditions for these transforms. Sections 4 and 5 are devoted to obtain these transforms of some standard functions. Some properties like Linearity, Scalar Multiplication, and Scaling are proved in sections 6 and 7. Section 8 is devoted to Discussion and Conclusion.

\section{RAM Finite Hyperbolic Transforms:}

Definition 2.1: Let $p \in C$ and $T$ be a finite number which may be positive or negative and $f(t)$ is a continuous or an almost piecewise continuous function defined over the interval $(0, T)$. Then RAM Finite Sine Hyperbolic transform of $f(t)$ is denoted by $R_{s h}(f(t))=F_{S}(p, T)$, and defined by

$\mathrm{R}_{\mathrm{sh}}(\mathrm{f}(\mathrm{t}))=\mathrm{F}_{\mathrm{S}}(\mathrm{p}, \mathrm{T})=\int_{0}^{\mathrm{T}} \sinh (\mathrm{pt}) \mathrm{f}(\mathrm{t}) \mathrm{dt}$,

where $\sinh (\mathrm{pt})$ is a Kernel of $\mathrm{R}_{\mathrm{sh}}$.

Here $\mathrm{R}_{\mathrm{sh}}$ is called RAM Finite Sine Hyperbolic transformation operator.

Definition 2.2: Let $p \in C$ and $T$ be a finite number which may be positive or negative and $f(t)$ is a continuous or an almost piecewise continuous function defined over the interval $(0, T)$. Then RAM Finite Cosine Hyperbolic transform of $f(t)$ is denoted by $R_{c h}(f(t))=F_{C}(p, T)$, and defined by

$R_{c h}(f(t))=F_{C}(p, T)=\int_{0}^{T} \cosh (p t) f(t) d t$,

where $\cosh (\mathrm{pt})$ is a Kernel of $\mathrm{R}_{\mathrm{ch}}$.

Here $\mathrm{R}_{\mathrm{ch}}$ is called RAM Finite Cosine Hyperbolic transformation operator.

Note : sinht, cosht are bounded for any bounded interval $\left(-\mathrm{T}_{1}, \mathrm{~T}_{2}\right)$.

\section{Existence of $\mathbf{R}_{\text {sh }}$ and $\mathbf{R}_{\text {ch. }}$.}

Theorem 3.1 If $f(t)$ is a piecewise continuous and absolutely integrable function on $(0, T)$, then $R_{s h}(f(t))$ exists. Proof: As sinht is bounded on $(0, T)$, there exist $K \in[0, \infty)$ such that $|\sinh (p t)| \leq K$ on $(0, T)$. Since $f(t)$ is absolutely integrable, there exist $\mathrm{M} \in[0, \infty)$ such that $\int_{0}^{\mathrm{T}}|\mathrm{f}(\mathrm{t})| \mathrm{dt} \leq \mathrm{M}$. 
Consider

$$
\begin{aligned}
\left|\mathrm{R}_{\text {sh }}(\mathrm{f}(\mathrm{t}))\right| & \quad\left|\int_{0}^{\mathrm{T}} \sinh (\mathrm{pt}) \mathrm{f}(\mathrm{t}) \mathrm{dt}\right| \\
& \leq \quad \int_{0}^{\mathrm{T}}|\sinh (\mathrm{pt})||\mathrm{f}(\mathrm{t})||\mathrm{dt}| \\
& \leq \quad \int_{0}^{\mathrm{T}} \mathrm{K}|\mathrm{f}(\mathrm{t}) \mathrm{dt}| \\
\Rightarrow \mid \mathrm{R}_{\text {sh }}(\mathrm{f}(\mathrm{t}) \mid & \leq \mathrm{K} \int_{0}^{\mathrm{T}}|\mathrm{f}(\mathrm{t}) \mathrm{dt}|
\end{aligned}
$$

Thus $\mathrm{R}_{\mathrm{sh}}(\mathrm{f}(\mathrm{t}))$ exists. Hence proved.

Theorem 3.2 If $f(t)$ is a piecewise continuous and absolutely integrable function on $(0, T)$, then $R_{c h}(f(t))$ exists. Proof: Consider

$$
\begin{aligned}
&\left|\mathrm{R}_{\mathrm{ch}}(\mathrm{f}(\mathrm{t}))\right|=\left|\int_{0}^{\mathrm{T}} \cosh (\mathrm{pt}) \mathrm{f}(\mathrm{t}) \mathrm{dt}\right| \\
& \leq \int_{0}^{\mathrm{T}}|\cosh (\mathrm{pt})| \mathrm{f}(\mathrm{t})|| \mathrm{dt} \mid \\
& \leq \int_{0}^{\mathrm{T}} \mathrm{K}|\mathrm{f}(\mathrm{t}) \mathrm{dt}| \quad(\operatorname{since}|\cosh (\mathrm{pt})| \leq \mathrm{K} \text { on }(0, \mathrm{~T}), 0 \leq \mathrm{K}<\infty) \\
& \leq \int_{0}^{\mathrm{T}}|\mathrm{f}(\mathrm{t}) \mathrm{dt}| \\
&\left.\Rightarrow\left|\mathrm{R}_{\mathrm{ch}}(\mathrm{f}(\mathrm{t}))\right| \leq \mathrm{M} . \mathrm{K} . \text { (since } \int_{0}^{\mathrm{T}}|\mathrm{f}(\mathrm{t})| \mathrm{dt} \leq \mathrm{M}, 0 \leq \mathrm{M}<\infty\right)
\end{aligned}
$$

Thus $\mathrm{R}_{\mathrm{ch}}(\mathrm{f}(\mathrm{t}))$ exists. Hence proved.

Theorem 3.3: If $f(t)$ is a piecewise continuous and bounded function on $(0, T)$, then Proof: Consider $\mathrm{R}_{\mathrm{sh}}(\mathrm{f}(\mathrm{t}))$ exists.

$$
\begin{aligned}
&\left|\mathrm{R}_{\mathrm{sh}}(\mathrm{f}(\mathrm{t}))\right|=\quad\left|\int_{0}^{\mathrm{T}} \sinh (\mathrm{pt}) \mathrm{f}(\mathrm{t}) \mathrm{dt}\right| \\
& \leq \quad \int_{0}^{\mathrm{T}}|\sinh (\mathrm{pt})||\mathrm{f}(\mathrm{t})||\mathrm{dt}| \\
& \leq \int_{0}^{\mathrm{T}} \text { M.K. }|\mathrm{dt}| \quad(\text { since }|\mathrm{f}(\mathrm{t})| \leq \mathrm{M} \text { on }(0, \mathrm{~T}), 0 \leq \mathrm{K}, \mathrm{M}<\infty) \\
& \Rightarrow \quad\left|\mathrm{R}_{\mathrm{sh}}(\mathrm{f}(\mathrm{t}))\right| \quad \leq \mathrm{M} . \mathrm{K} . \mathrm{T} .
\end{aligned}
$$

Thus $\mathrm{R}_{\mathrm{sh}}$ exists. Hence proved.

Theorem 3.4: If $f(t)$ is a piecewise continuous and bounded function on $(0, T)$, then $R_{c h}(f(t))$ exists.

Proof : consider

$$
\begin{aligned}
\left|\mathrm{R}_{\mathrm{sh}}(\mathrm{f}(\mathrm{t}))\right| & =\left|\int_{0}^{\mathrm{T}} \cosh (\mathrm{pt}) \mathrm{f}(\mathrm{t}) \mathrm{dt}\right| \\
& \leq \quad \int_{0}^{\mathrm{T}}|\cosh (\mathrm{pt})||\mathrm{f}(\mathrm{t}) \mathrm{dt}| \\
& \leq \mathrm{MK} \int_{0}^{T}|\mathrm{dt}|(\operatorname{since}|\cosh (\mathrm{pt})| \leq \mathrm{K},|\mathrm{f}(\mathrm{t})| \leq \mathrm{M} \text { on }(0, \mathrm{~T}), 0 \leq \mathrm{K}, \mathrm{M}<\infty) \\
\Rightarrow\left|\mathrm{R}_{\mathrm{ch}}(\mathrm{f}(\mathrm{t}))\right| & \leq \mathrm{M} \cdot \text { K.T. }
\end{aligned}
$$

Thus $R_{c h}(f(t))$ exists. Hence Proved.

\section{RAM Finite Sine Hyperbolic transform of some standard functions:}


1. $\quad \mathrm{R}_{\mathrm{sh}}(1)=\frac{\cosh (p T)-1}{p}$

Proof:

$\begin{aligned} \mathrm{R}_{\mathrm{sh}}(1) & =\int_{0}^{\mathrm{T}} \sinh (\mathrm{pt}) \mathrm{dt} \\ & =\frac{\cosh (p T)-1}{p}\end{aligned}$

2. $\quad \mathrm{R}_{\mathrm{sh}}(\mathrm{t})=\frac{T \cosh (p \mathrm{~T})}{p}-\frac{\sinh (p \mathrm{~T})}{p^{2}}$

Proof:

$\begin{aligned} \mathrm{R}_{\mathrm{sh}}(\mathrm{t}) & =\int_{0}^{\mathrm{T}} \mathrm{t} \sinh (\mathrm{pt}) \mathrm{dt} \\ & =\frac{T \cosh (p \mathrm{~T})}{p}-\frac{\sinh (p \mathrm{~T})}{p^{2}}\end{aligned}$

3. $\mathrm{R}_{\mathrm{sh}}\left(\mathrm{t}^{2}\right)=\frac{T^{2} \cosh (p \mathrm{~T})}{p}-\frac{2 T \sinh (p \mathrm{~T})}{p^{2}}+\frac{(2 \cosh (p T)-2)}{\mathrm{p}^{3}}$.

Proof:

$$
\begin{gathered}
\mathrm{R}_{\mathrm{sh}}\left(\mathrm{t}^{2}\right) \quad=\quad \int_{0}^{\mathrm{T}} \mathrm{t}^{2} \sinh (\mathrm{pt}) \mathrm{dt} \\
\quad=\quad \frac{T^{2} \cosh (p \mathrm{~T})}{p}-\frac{2 T \sinh (p \mathrm{~T})}{p^{2}}+\frac{(2 \cosh (p T)-2)}{\mathrm{p}^{3}} . \\
\text { 4. } \quad \mathrm{R}_{\mathrm{sh}}\left(\mathrm{t}^{\mathrm{k}}\right)=\left\{\begin{array}{l}
\frac{\mathrm{T}^{k} \cosh (p T)}{p}-\frac{k T^{k-1} \sinh (p T)}{P^{2}}+\ldots .+\frac{k !(-1)^{k}[\cosh (p T)-1]}{p^{k}}, \text { if } \mathrm{k} \text { is even } \\
\frac{\mathrm{T}^{k} \cosh (p T)}{p}-\frac{k T^{k-1} \sinh (p T)}{P^{2}}+\ldots .+\frac{k !(-1)^{k} \sinh (p T)}{p^{k}}, \text { if } \mathrm{k} \text { is odd }
\end{array}\right.
\end{gathered}
$$

Proof:

$$
\begin{aligned}
& \mathrm{R}_{\mathrm{sh}}\left(\mathrm{t}^{\mathrm{k}}\right)=\quad \int_{0}^{\mathrm{T}} \mathrm{t}^{\mathrm{k}} \sinh (\mathrm{pt}) \mathrm{dt} \\
& =\left\{\begin{array}{l}
{\left[\frac{t^{k} \cosh (p t)}{p}\right]_{0}^{T}-\left[\frac{k t^{k-1} \sinh (p t)}{p^{2}}\right]_{0}^{T}+\ldots \ldots .+\left[\frac{k !(-1)^{k} \cosh (p t)}{p^{k}}\right]_{0}^{T}, \text { if } \mathrm{k} \text { is even, }} \\
{\left[\frac{t^{k} \cosh (p t)}{p}\right]_{0}^{T}-\left[\frac{k t^{k-1} \sinh (p t)}{p^{2}}\right]_{0}^{T}+\ldots \ldots .+\left[\frac{k !(-1)^{k} \sinh (p t)}{p^{k}}\right]_{0}^{T}, \text { if } \mathrm{k} \text { is odd. }}
\end{array}\right. \\
& =\left\{\begin{array}{l}
\frac{\mathrm{T}^{k} \cosh (p T)}{p}-\frac{k T^{k-1} \sinh (p T)}{P^{2}}+\ldots .+\frac{k !(-1)^{k}[\cosh (p T)-1]}{p^{k}}, \text { if } \mathrm{k} \text { is even } \\
\frac{\mathrm{T}^{k} \cosh (p T)}{p}-\frac{k T^{k-1} \sinh (p T)}{P^{2}}+\ldots .+\frac{k !(-1)^{k} \sinh (p T)}{p^{k}}, \text { if } \mathrm{k} \text { is odd }
\end{array}\right.
\end{aligned}
$$

5. $\quad \mathrm{R}_{\mathrm{sh}}(\sin (\mathrm{at})) \quad=\left(\frac{-a}{p^{2}+a^{2}}\right) \sinh (\mathrm{pT}) \cos (\mathrm{aT})+\left(\frac{p}{p^{2}+a^{2}}\right) \cosh (\mathrm{pT}) \sin (\mathrm{aT})$.

Proof: 


$$
\begin{aligned}
& \mathrm{R}_{\mathrm{sh}}(\sin (\mathrm{at}))=\int_{0}^{\mathrm{T}} \sin (\mathrm{at}) \sinh (\mathrm{pt}) \mathrm{dt} \\
&=\quad \frac{\sinh (p T) \cos (a T)}{-a}+\frac{p \cos (p T) \sin (a T)}{a^{2}}-\frac{p^{2} R_{s h}(\sin (a t)}{a^{2}} . \\
& \Rightarrow\left(1+\frac{p^{2}}{a^{2}}\right) \mathrm{R}_{\mathrm{sh}}(\sin (\mathrm{at})) \quad=\frac{\sinh (p T) \cdot \cos (a T)}{-a}+\frac{p \cdot \cos (p T) \cdot \sin (a T)}{a^{2}}
\end{aligned}
$$

i.e. $\mathrm{R}_{\mathrm{sh}}(\sin (\mathrm{at}))=\left(\frac{-a}{p^{2}+a^{2}}\right) \sinh (\mathrm{pT}) \cdot \cos (\mathrm{aT})+\left(\frac{p}{p^{2}+a^{2}}\right) \cosh (\mathrm{pT}) \sin (\mathrm{aT}) \cdot$

6. $\mathrm{R}_{\mathrm{sh}}(\cos (\mathrm{at}))=\left(\frac{a}{p^{2}+a^{2}}\right) \sinh (\mathrm{pT}) \cdot \sin (\mathrm{aT})+\left(\frac{p}{p^{2}+a^{2}}\right)[\cosh (\mathrm{pT}) \cdot \cos (\mathrm{aT})-1] \cdot$

Proof:

$$
\begin{gathered}
\mathrm{R}_{\mathrm{sh}}(\cos (\mathrm{at})) \quad=\int_{0}^{\mathrm{T}} \cos (\mathrm{at}) \sinh (\mathrm{pt}) \mathrm{dt} \\
=\frac{\sinh (p T) \cdot \sin (a T)}{a}+\frac{[p \cdot \cosh (p T) \cdot \cos (a T)-p]}{a^{2}}-\frac{p^{2} \cdot R_{s h}(\cos (a t))}{a^{2}} \\
\Rightarrow\left(1+\frac{p^{2}}{a^{2}}\right) \mathrm{R}_{\mathrm{sh}}(\cos (\mathrm{at}))=\frac{\sinh (p T) \sin (a T)}{a}+\frac{[p \cdot \cosh (p T) \cdot \cos (a T)-p]}{a^{2}} \\
\text { i.e. } \mathrm{R}_{\mathrm{sh}}(\cos (\mathrm{at}))=\left(\frac{a}{p^{2}+a^{2}}\right) \sinh (\mathrm{pT}) \cdot \sin (\mathrm{aT})+\left(\frac{p}{p^{2}+a^{2}}\right)[\cosh (\mathrm{pT}) \cdot \cos (\mathrm{aT})-1] . \\
\text { 7. } \mathrm{R}_{\mathrm{sh}}\left(\mathrm{e}^{\mathrm{at}}\right)=\left(\frac{-a}{p^{2}-a^{2}}\right) \sinh (\mathrm{pT}) \cdot \mathrm{e}^{\mathrm{aT}}+\left(\frac{p}{p^{2}-a^{2}}\right)\left[\cosh (\mathrm{pT}) \cdot \mathrm{e}^{\mathrm{aT}}-1\right], \operatorname{provided} \mathrm{p}^{2} \neq \mathrm{a}^{2} .
\end{gathered}
$$

Proof:

$$
\begin{gathered}
\mathrm{R}_{\mathrm{sh}}\left(\mathrm{e}^{\mathrm{at}}\right)=\int_{0}^{\mathrm{T}} \quad \mathrm{e}^{\mathrm{at}} \sinh (\mathrm{pt}) \mathrm{dt} \\
=\frac{\sinh (p T) \cdot e^{a T}}{a}-\frac{\left[p \cdot \cos (p T) e^{a T}-p\right]}{a^{2}}+\frac{p^{2} \cdot R_{s h}\left(e^{a t}\right)}{a^{2}} \\
\Rightarrow \mathrm{R}_{\mathrm{sh}}\left(\mathrm{e}^{\mathrm{at}}\right)=\left(\frac{-a}{p^{2}-a^{2}}\right)-\sinh (\mathrm{pT}) \cdot \mathrm{e}^{\mathrm{aT}}+\left(\frac{p}{p^{2}-a^{2}}\right)\left[\cosh (\mathrm{pT}) \cdot \mathrm{e}^{\mathrm{aT}}-1\right], \text { provided } \mathrm{p}^{2} \neq \mathrm{a}^{2} . \\
\text { 8. } \mathrm{R}_{\text {sh }}\left(\mathrm{e}^{-\mathrm{at}}\right)=\left(\frac{a}{p^{2}-a^{2}}\right) \sinh (\mathrm{pT}) \cdot \mathrm{e}^{-\mathrm{aT}}+\left(\frac{-p}{p^{2}-a^{2}}\right)\left[1-\cosh (\mathrm{pT}) \cdot \mathrm{e}^{-\mathrm{aT}}\right], \text { Provided } \mathrm{P}^{2} \neq \mathrm{a}^{2} .
\end{gathered}
$$

Proof:

$$
\begin{aligned}
& \mathrm{R}_{\mathrm{sh}}\left(\mathrm{e}^{-\mathrm{at}}\right)=\int_{0}^{\mathrm{T}} \mathrm{e}^{\mathrm{-at}} \sinh (\mathrm{pt}) \mathrm{dt} \\
& =\frac{\sinh (p T) \cdot e^{-a T}}{-a}-\frac{\left[p \cdot \cosh (p T) \cdot e^{-a T}-p\right]}{a^{2}}+\frac{p^{2} R_{s h}\left(e^{-a t}\right)}{a^{2}} \\
& \Rightarrow\left(1-\frac{p^{2}}{a^{2}}\right) \mathrm{R}_{\mathrm{sh}}\left(\mathrm{e}^{-\mathrm{at}}\right)=\frac{\sinh (p T) \cdot e^{-a T}}{-a}-\frac{\left[p \cdot \cosh (p T) \cdot e^{-a T}-p\right]}{a^{2}} \\
& \text { i.e. } \mathrm{R}_{\mathrm{sh}}\left(\mathrm{e}^{-\mathrm{at}}\right)=\left(\frac{a}{p^{2}-a^{2}}\right) \sinh (\mathrm{pT}) \cdot \mathrm{e}^{-\mathrm{aT}}+\left(\frac{-p}{p^{2}-a^{2}}\right)\left[1-\cosh (\mathrm{pT}) \cdot \mathrm{e}^{-\mathrm{aT}}\right] \text {, provided } \mathrm{p}^{2} \neq \mathrm{a}^{2} \text {. }
\end{aligned}
$$




\section{RAM Finite Cosine Hyperbolic Transform of some standard functions:}

1. $\mathrm{R}_{\mathrm{ch}}(1)=\frac{\sinh (p T)}{p}$.

Proof:

$$
\begin{aligned}
\mathrm{R}_{\mathrm{ch}}(\mathrm{t}) & =\int_{0}^{\mathrm{T}} \cosh (\mathrm{pt}) \mathrm{dt} \\
& =\frac{\sinh (p T)}{p}
\end{aligned}
$$

2. $\quad \mathrm{R}_{\mathrm{ch}}(\mathrm{t})=\frac{\mathrm{T} \sinh (\mathrm{pT})}{p}-\left(\frac{\cosh (p T)-1}{p^{2}}\right)$

Proof:

$$
\begin{aligned}
\mathrm{R}_{\mathrm{ch}}(\mathrm{t}) & =\int_{0}^{\mathrm{T}} \mathrm{t} \cosh (\mathrm{pt}) \mathrm{dt} \\
& =\frac{\mathrm{T} \sinh (\mathrm{pT})}{p}-\left(\frac{\cosh (p T)-1}{p^{2}}\right)
\end{aligned}
$$

3. $\mathrm{R}_{\mathrm{ch}}\left(\mathrm{t}^{2}\right)=\frac{T^{2} \cdot \sinh (p T)}{p}-\frac{2 \cdot T \cdot \cosh (p T)}{P^{2}}+\frac{2 \cdot \sinh (p T)}{p^{3}}$.

Proof:

$$
\begin{gathered}
\mathrm{R}_{\mathrm{ch}}(\mathrm{t})=\quad \int_{0}^{\mathrm{T}} \mathrm{t}^{2} \cdot \cosh (\mathrm{pt}) \mathrm{dt} \\
=\quad \frac{T^{2} \cdot \sinh (p T)}{p}-\frac{2 \cdot T \cdot \cosh (p T)}{P^{2}}+\frac{2 \cdot \sinh (p T)}{p^{3}} . \\
\text { 4. } \quad \mathrm{R}_{\mathrm{ch}}\left(\mathrm{t}^{\mathrm{k}}\right)=\left\{\begin{array}{l}
\frac{\mathrm{T}^{k} \sinh (p T)}{p}-\frac{k T^{k-1} \cosh (p T)}{p^{2}}+\ldots \ldots .+\frac{k !(-1)^{k} \sinh (p T)}{p^{k}}, \text { if } \mathrm{k} \text { is even, } \\
\frac{T^{k} \sinh (p T)}{p}-\frac{k T^{k-1} \cosh (p T)}{p^{2}}+\ldots \ldots .+\frac{k !(-1)^{k}[\cosh (p T)-1]}{p^{k}}, \text { if } \mathrm{k} \text { is odd. }
\end{array}\right.
\end{gathered}
$$

Proof:

$\mathrm{R}_{\mathrm{ch}}\left(\mathrm{t}^{\mathrm{k}}\right)=\int_{0}^{\mathrm{T}} \mathrm{t}^{\mathrm{k}} \cosh (\mathrm{pt}) \mathrm{dt}$ $=\left\{\begin{array}{l}{\left[\frac{\mathrm{t}^{k} \sinh (p t)}{p}\right]_{0}^{T}-\left[\frac{k t^{k-1} \cosh (p T)}{P^{2}}\right]_{0}^{T}+\ldots .+\left[\frac{k !(-1)^{k} \sinh (p t)}{p^{k}}\right]_{0}^{T}, \text { if } \mathrm{k} \text { is even }} \\ {\left[\frac{\mathrm{t}^{k} \cdot \sinh (p t)}{p}\right]_{0}^{T}-\left[\frac{k t^{k-1} \cosh (p t)}{P^{2}}\right]_{0}^{T}+\ldots .+\left[\frac{k !(-1)^{k} \cosh (p t)}{p^{k}}\right]_{0}^{T}, \text { if } \mathrm{k} \text { is odd }}\end{array}\right.$ $=\left\{\begin{array}{l}\frac{\mathrm{T}^{k} \sinh (p T)}{p}-\frac{k T^{k-1} \cosh (p T)}{p^{2}}+\ldots \ldots .+\frac{k !(-1)^{k} \sinh (p T)}{p^{k}}, \text { if } \mathrm{k} \text { is even, } \\ \frac{T^{k} \sinh (p T)}{p}-\frac{k T^{k-1} \cosh (p T)}{p^{2}}+\ldots \ldots .+\frac{k !(-1)^{k}[\cosh (p T)-1]}{p^{k}}, \text { if } \mathrm{k} \text { is odd. }\end{array}\right.$

5. $\quad \mathrm{R}_{\mathrm{ch}}(\operatorname{Sin}(\mathrm{at}))=\left(\frac{a}{p^{2}+a^{2}}\right)[1-\cosh (\mathrm{pT}) \cos (\mathrm{aT})]+\left(\frac{p}{p^{2}+a^{2}}\right) \sinh (\mathrm{pT}) \sin (\mathrm{aT})$. 


\section{Proof:}

$$
\begin{aligned}
& \mathrm{R}_{\mathrm{ch}}(\sin (\mathrm{at}))=\int_{0}^{\mathrm{T}} \sin (\mathrm{at}) \cosh (\mathrm{pt}) \mathrm{dt} \\
& =\frac{[\cos (p T) \cos (a T)-1]}{-a}+\frac{p \sinh (p T) \sin (a T)}{a^{2}}-\frac{p^{2} R_{c h}(\sin (a t))}{a^{2}} \\
& \Rightarrow\left(1+\frac{p^{2}}{a^{2}}\right) \mathrm{R}_{\mathrm{ch}}(\sin (\mathrm{at})) \quad=\frac{[\cos (p T) \cos (a T)-1]}{-a}+\frac{p \sinh (p T) \sin (a T)}{a^{2}} \\
& \text { i.e. } \mathrm{R}_{\mathrm{ch}}(\operatorname{Sin}(\mathrm{at})) \quad=\left(\frac{a}{p^{2}+a^{2}}\right)[1-\cosh (\mathrm{pT}) \cos (\mathrm{aT})]+\left(\frac{p}{p^{2}+a^{2}}\right) \sinh (\mathrm{pT}) \sin (\mathrm{aT}) \text {. } \\
& \text { 6. } \quad \mathrm{R}_{\mathrm{ch}}(\cos (\mathrm{at}))=\left(\frac{a}{p^{2}+a^{2}}\right) \cosh (\mathrm{pT}) \sin (\mathrm{aT})+\left(\frac{p}{p^{2}+a^{2}}\right) \sinh (\mathrm{pT}) \cos (\mathrm{aT}) \text {. }
\end{aligned}
$$

\section{Proof:}

$$
\begin{aligned}
& \mathrm{R}_{\mathrm{ch}} \cos (\mathrm{at})=\int_{0}^{\mathrm{T}} \cos (\mathrm{at}) \cosh (\mathrm{pt}) \mathrm{dt} \\
&=\frac{\cosh (p T) \sin (a T)}{a}+\frac{p \sinh (p T) \cos (a T)}{a^{2}}-\frac{p^{2} R_{c h}(\cos (a t))}{a^{2}} . \\
& \Rightarrow \quad\left(1+\frac{p^{2}}{a^{2}}\right) \mathrm{R}_{\mathrm{ch}}(\cos (\mathrm{at})) \quad=\frac{\cosh (p T) \sin (a T)}{a}+\frac{p \sinh (p T) \cos (a T)}{a^{2}}
\end{aligned}
$$

i.e. $\mathrm{R}_{\mathrm{ch}}(\cos (\mathrm{at}))=\left(\frac{a}{p^{2}+a^{2}}\right) \cosh (\mathrm{pT}) \sin (\mathrm{aT})+\left(\frac{p}{p^{2}+a^{2}}\right) \sinh (\mathrm{pT}) \cos (\mathrm{aT})$.

7. $\quad \mathrm{R}_{\mathrm{ch}}\left(\mathrm{e}^{\mathrm{at}}\right)=\left(\frac{a}{p^{2}-a^{2}}\right)\left[\cosh (\mathrm{pT}) \mathrm{e}^{\mathrm{aT}}-1\right]+\left(\frac{p}{p^{2}-a^{2}}\right) \sinh (\mathrm{pT}) \mathrm{e}^{\mathrm{aT}}, \operatorname{Provided} \mathrm{p}^{2} \neq \mathrm{a}^{2}$.

Proof:

$$
\begin{gathered}
\mathrm{R}_{\mathrm{ch}}\left(\mathrm{e}^{\mathrm{at}}\right)=\quad \int_{0}^{\mathrm{T}} \mathrm{e}^{\mathrm{at}} \cosh (\mathrm{pt}) \mathrm{dt} \\
=\quad\left(1-\frac{p^{2}}{a^{2}}\right) \mathrm{R}_{\mathrm{ch}}\left(\mathrm{e}^{\mathrm{at}}\right) \quad=\frac{\left[\cosh (p T) e^{a T}-1\right]}{a}-\frac{p \cdot \sinh (p T) e^{a T}}{a^{2}}+\frac{p^{2} R_{c h}\left(e^{a t}\right)}{a^{2}} . \\
\Rightarrow \quad \text { i.e. } \mathrm{R}_{\mathrm{ch}}\left(\mathrm{e}^{\mathrm{at}}\right)=\left(\frac{a}{p^{2}-a^{2}}\right)\left[\cosh (\mathrm{pT}) \mathrm{e}^{\mathrm{aT}}-1\right]+\left(\frac{\left.p \cosh (p) e^{a T}-1\right]}{p^{2}-a^{2}}\right) \sinh (\mathrm{pT}) \mathrm{e}^{\mathrm{aT}}, \operatorname{Provided} \mathrm{p}^{2} \neq \mathrm{a}^{2} . \\
\text { 8. } \mathrm{R}_{\mathrm{ch}}\left(\mathrm{e}^{-\mathrm{at}}\right) \quad\left(\frac{a}{p^{2}-a^{2}}\right) \cosh (\mathrm{pT}) \mathrm{e}^{-\mathrm{aT}}+\left(\frac{-p}{p^{2}-a^{2}}\right)\left[1-\sinh (\mathrm{pT}) \mathrm{e}^{-\mathrm{aT}}\right], \operatorname{provided} \mathrm{p}^{2} \neq \mathrm{a}^{2} .
\end{gathered}
$$

\section{Proof :}

$$
\begin{aligned}
\mathrm{R}_{\mathrm{ch}}\left(\mathrm{e}^{-\mathrm{at}}\right) & =\int_{0}^{\mathrm{T}} \mathrm{e}^{-\mathrm{at}} \cosh (\mathrm{pt}) \mathrm{dt} \\
& =\frac{\left[\cosh (p T) e^{-a T}-1\right]}{-a}-\frac{p \sinh (p T) e^{-a T}}{a^{2}}+\frac{p^{2} R_{c h}\left(e^{-a t}\right)}{a^{2}} \\
\Rightarrow \quad & \left(1-\frac{p^{2}}{a^{2}}\right) \mathrm{R}_{\mathrm{ch}}\left(\mathrm{e}^{-\mathrm{at}}\right) \quad=\frac{\left[\cosh (p T) e^{-a T}-1\right]}{-a}-\frac{p \sinh (p T) e^{-a T}}{a^{2}}
\end{aligned}
$$


i.e. $\mathrm{R}_{\text {ch }}\left(\mathrm{e}^{-\mathrm{at}}\right) \quad=\left(\frac{a}{p^{2}-a^{2}}\right)\left(\cosh (\mathrm{pT}) \mathrm{e}^{-\mathrm{aT}}-1\right)+\left(\frac{-p}{p^{2}-a^{2}}\right)\left[\sinh (\mathrm{pT}) \mathrm{e}^{-\mathrm{aT}}\right] ; \operatorname{provided} \mathrm{p}^{2} \neq \mathrm{a}^{2}$.

VI. Some Properties of RAM Finite Sine Hyperbolic transform:

1. Linearity: $\quad \mathrm{R}_{\mathrm{sh}}\left(\mathrm{f}_{1}(\mathrm{t})+\mathrm{f}_{2}(\mathrm{t})\right)=\mathrm{R}_{\mathrm{sh}}\left(\mathrm{f}_{1}(\mathrm{t})\right)+\mathrm{R}_{\mathrm{sh}}\left(\mathrm{f}_{2}(\mathrm{t})\right)$.

Proof: Let $0<\mathrm{t}<\mathrm{T}$, then by definition

$$
\begin{array}{rlrl}
\mathrm{R}_{\mathrm{sh}}\left(\mathrm{f}_{1}(\mathrm{t})+\mathrm{f}_{2}(\mathrm{t})\right) & = & \int_{0}^{\mathrm{T}}\left(\mathrm{f}_{1}(\mathrm{t})+\mathrm{f}_{2}(\mathrm{t})\right) \sinh (\mathrm{pt}) \mathrm{dt} \\
& =\int_{0}^{\mathrm{T}} \mathrm{f}_{1}(\mathrm{t}) \sinh (\mathrm{pt}) \mathrm{dt}+\int_{0}^{\mathrm{T}} \mathrm{f}_{2}(\mathrm{t}) \sinh (\mathrm{pt}) \mathrm{dt} \\
& =\quad \mathrm{R}_{\mathrm{sh}}\left(\mathrm{f}_{1}(\mathrm{t})\right)+\mathrm{R}_{\mathrm{sh}}\left(\mathrm{f}_{2}(\mathrm{t})\right) .
\end{array}
$$

2. Scalar Multiplication: If $\mathrm{c}$ be any constant, then $\mathrm{R}_{\mathrm{sh}}(\mathrm{cf}(\mathrm{t}))=\mathrm{cR}_{\mathrm{sh}}(\mathrm{f}(\mathrm{t}))$.

Proof: Let $\mathrm{c}$ be any constant, then by definition

$$
\begin{aligned}
\mathrm{R}_{\mathrm{sh}}(\mathrm{cf}(\mathrm{t})) & =\int_{0}^{\mathrm{T}} \mathrm{cf}(\mathrm{t}) \sinh (\mathrm{pt}) \mathrm{dt} \\
& =\quad \mathrm{c} \int_{0}^{\mathrm{T}} \mathrm{f}(\mathrm{t}) \sinh (\mathrm{pt}) \mathrm{dt} \\
& =\quad \mathrm{cR}_{\mathrm{sh}}(\mathrm{f}(\mathrm{t})) .
\end{aligned}
$$

3. Scaling: If $\mathrm{R}_{\mathrm{sh}}(\mathrm{f}(\mathrm{t}))=\mathrm{F}_{\mathrm{S}}(\mathrm{p}, \mathrm{T})$ then $\mathrm{R}_{\mathrm{sh}}(\mathrm{f}(\mathrm{at}))=\frac{F_{S}\left(\frac{p}{a}, a T\right)}{a}$

Proof: Let $R_{s h}(f(t))=F_{S}(p, T)$, then by definition

$$
\begin{aligned}
\mathrm{R}_{\mathrm{sh}}(\mathrm{f}(\mathrm{at})) & =\int_{0}^{\mathrm{T}} \mathrm{f}(\mathrm{at}) \sinh (\mathrm{pt}) \mathrm{dt} \\
& =\int_{0}^{\mathrm{T}} \frac{f(x) \sinh \left(\frac{x p}{a}\right)}{a} d x \\
& =\frac{F_{S}\left(\frac{p}{a}, a T\right)}{a}
\end{aligned}
$$

VII. Some Properties of RAM Finite Cosine Hyperbolic transform:

1. $\quad$ Linearity $: R_{c h}\left(f_{1}(t)+f_{2}(t)\right)=R_{c h}\left(f_{1}(t)\right)+R_{c h}\left(f_{2}(t)\right)$

Proof: Let $0<\mathrm{t}<\mathrm{T}$, then by definition

$$
\begin{aligned}
\mathrm{R}_{\mathrm{ch}}\left(\mathrm{f}_{1}(\mathrm{t})+\mathrm{f}_{2}(\mathrm{t})\right) & =\int_{0}^{T}\left(\mathrm{f}_{1}(\mathrm{t})+\mathrm{f}_{2}(\mathrm{t})\right) \cosh (\mathrm{pt}) \mathrm{dt} \\
& =\int_{0}^{T} \mathrm{f}_{1}(\mathrm{t}) \cosh (\mathrm{pt}) \mathrm{dt}+\int_{0}^{T} \mathrm{f}_{2}(\mathrm{t}) \cosh (\mathrm{pt}) \mathrm{dt} \\
& =\mathrm{R}_{\mathrm{ch}}\left(\mathrm{f}_{1}(\mathrm{t})\right)+\mathrm{R}_{\mathrm{ch}}\left(\mathrm{f}_{2}(\mathrm{t})\right) .
\end{aligned}
$$

2. Scalar Multiplication: If $\mathrm{c}$ is any constant, then $\mathrm{R}_{\mathrm{ch}}(\mathrm{c} . \mathrm{f}(\mathrm{t}))=\mathrm{c} \cdot \mathrm{R}_{\mathrm{ch}}(\mathrm{f}(\mathrm{t}))$

Proof: Let $\mathrm{c}$ be any constant, then by definition

$$
\begin{aligned}
\mathrm{R}_{\mathrm{ch}}(\mathrm{cf}(\mathrm{t})) & =\int_{0}^{T}(\mathrm{cf}(\mathrm{t})) \cosh (\mathrm{pt}) \mathrm{dt} \\
& =\mathrm{c} \int_{0}^{T} \mathrm{f}(\mathrm{t}) \cosh (\mathrm{pt}) \mathrm{dt} \\
& =\mathrm{c} \mathrm{R}_{\mathrm{ch}}(\mathrm{f}(\mathrm{t})) .
\end{aligned}
$$


3. Scaling: If $\mathrm{R}_{\mathrm{ch}}(\mathrm{f}(\mathrm{t}))=\mathrm{F}_{\mathrm{C}}(\mathrm{p}, \mathrm{T})$, then $\mathrm{R}_{\mathrm{ch}}(\mathrm{f}(\mathrm{at}))=\frac{F_{C}\left(\frac{p}{a}, a T\right)}{a}$

Proof: Let $R_{c h}(f(t))=F_{C}(p, T)$, then

$$
\begin{aligned}
\mathrm{R}_{\mathrm{ch}}(\mathrm{f}(\mathrm{at})) & =\int_{0}^{T} \mathrm{f}(\mathrm{at}) \cosh (\mathrm{pt}) \mathrm{dt} \\
& =\frac{\int_{0}^{T}(f(x)) \cosh \left(\frac{x p}{a}\right)}{a} d x \\
& =\frac{F_{C}\left(\frac{p}{a}, a T\right)}{a}
\end{aligned}
$$

\section{Discussion and Conclusion:}

Unlike the usual Laplace transform of a function $\mathrm{f}(\mathrm{t})$, there is no restriction needed on the transform variable $p$ for the existence of $R_{c h}(f(t))$ and $R_{s h}(f(t))$. Further, the existence of $R_{c h}(f(t))$ and $R_{s h}(f(t))$ does not require exponential order property of a function $f(t)$. If a function $f(t)$ has the usual Laplace transform, then it also has the RAM Finite Sine Hyperbolic transform and RAM Finite Cosine Hyperbolic transform. In other words, if $\mathrm{L}(\mathrm{f}(\mathrm{t}))$ exists, then $\mathrm{R}_{\mathrm{ch}}(\mathrm{f}(\mathrm{t}))$ and $\mathrm{R}_{\mathrm{sh}}(\mathrm{f}(\mathrm{t})$ )exists as shown below. We have

$$
\begin{aligned}
\mathrm{L}(\mathrm{f}(\mathrm{t})) & =\int_{0}^{\infty} \mathrm{f}(\mathrm{t}) \mathrm{e}^{-\mathrm{pt}} \mathrm{dt} \\
& =\int_{0}^{T} \mathrm{f}(\mathrm{t}) \cosh (\mathrm{pt}) \mathrm{dt}-\int_{0}^{T} \mathrm{f}(\mathrm{t}) \sin (\mathrm{pt}) \mathrm{dt}+\int_{T}^{\infty} \mathrm{f}(\mathrm{t}) \mathrm{e}^{-\mathrm{pt}} \mathrm{dt} \\
& =\mathrm{R}_{\mathrm{ch}}(\mathrm{f}(\mathrm{t}))-\mathrm{R}_{\mathrm{sh}}(\mathrm{f}(\mathrm{t}))+\int_{T}^{\infty} \mathrm{f}(\mathrm{t}) \mathrm{e}^{-\mathrm{pt}} \mathrm{dt} .
\end{aligned}
$$

Since $L(f(t))$ exists, all the three integrals on R.H.S. exist. Hence, if $L(f(t))$ exists then $R_{c h}(f(t))$ and $\mathrm{R}_{\mathrm{sh}}(\mathrm{f}(\mathrm{t})$ )exists but converse is not necessarily true. This can be shown by an example. It is well known that the usual Laplace transform of $f(t)=e^{\text {at }}$, for $a>0$, does not exist but $R_{c h}\left(e^{\text {at }}\right)$ and $R_{s h}\left(e^{\text {at }}\right)$ both exists.

\section{References:}

[1]. Chandrasenkharan K., Classical Fourier Transform, Springer-Verlag, New York (1989).

[2]. Debnath L.and Thomas J., On Finite Laplace Transformation with Application, Z.Angrew.Math.und meth.56(1976),559-593.

[3]. S.B.Chavan, V.C.Borkar., "Canonical Sine transform and their Unitary Representation", Int. J. Contemp. Math. Science, Vol.7, 2012, No. 15,717-725

[4]. S.B.Chavan, V.C.Borkar., "Operation Calculus of Canonical Cosine transform", IAENG International Journal of Applied Mathematics, 2012.

[5]. S.B.Chavan, V.C.Borkar., "Some aspect of Canonical Cosine transform of generalised function", Bulletin of Pure and Applied Sciences.Vol.29E(No.1),2010.

[6]. S.B.Chavan, V.C.Borkar., "Analyticity and Inversion for Generalised Canonical Sine transforms", Applied Science Periodical Vol.XIV, (No 2), May 2012.

[7]. Lokenath debnath, Dombaru Bhatta, Integral Transfer and there application, Chapman and Hall/CRC, Taylor and Francis group, (2007).

[8]. Watson E.J., Laplace Transformation and Application, Van Nostrand, Reinhold, New York(1981).

[9]. S.B.Chavan, V.C.Borkar., "Some properties and Applications of Generalised Canonical Transforms", Inter National Journal of Applied Sciences, Vol. 5, No.7, 309-314, 2011.

[10]. Wyman M., The method of the Laplace Transformation, Roy.Soc.Canada,(2)(1964),227-256

[11]. Zemanian A.H., Generalised Integral Transformation, John Wiley and Son, New York, (1969).

[12]. Zemanian A.H., Distribution Theory and Transform Analysis, John Wiley and Son, New York, (1969). 